Notfall Rettungsmed $2021 \cdot 24: 887-888$ https://doi.org/10.1007/s10049-021-00931-8 Angenommen: 21. Juli 2021

Online publiziert: 16. August 2021

(c) Springer Medizin Verlag $\mathrm{GmbH}$, ein Teil von Springer Nature 2021

\section{Prof. Dr. Dr. h.c. Wolfgang F. Dick}

\section{Juli $1936-8$. Mai 2021}

\author{
Ines Wolff' · Bernd W. Böttiger ${ }^{2} \cdot$ Christian Waydhas ${ }^{3} \cdot$ Christian Wrede $^{4}$. \\ Paul Herrmann ${ }^{1}$ \\ ${ }^{1}$ Fachzeitschriften Medizin, Springer Medizin Verlag GmbH, Heidelberg, Deutschland \\ ${ }^{2}$ Klinik für Anästhesiologie und Operative Intensivmedizin, Köln, Deutschland \\ ${ }^{3}$ Chirurgische Universitätsklinik und Poliklinik, Berufsgenossenschaftliches Universitätsklinikum \\ Bergmannsheil, Bochum, Deutschland \\ ${ }^{4}$ Interdisziplinäres Notfallzentrum mit Rettungsstelle, Helios-Klinikum Berlin-Buch, Berlin, Deutschland
}

Im Rahmen der Herausgebersitzung der Notfall+Rettungsmedizin am 9. Juni 2021 haben wir, das Board der Zeitschrift und die Verlagsmitarbeiterinnen, erfahren, dass am Tag zuvor, am 8. Juni, mit 84 Jahren Herr Professor Dr. Dr. h.c. Wolfgang Dick verstorben ist.

\section{I) Wir nehmen Abschied vom Gründungsvater der Notfall+Rettungsmedizin}

Mit Trauer und Bestürzung haben wir gemeinsam auf der Sitzung in Stille von dem Gründungsvater der Notfall+Rettungsmedizin Abschied genommen.

Gemeinsam mit Professor Dr. Peter Lemburg und Professor Dr. Hans-Peter Schuster hat Professor Dick (D Abb. 1) im Jahr 1997 die Zeitschrift Notfall gegründet, die sich dann, ein Jahr später, zu Notfall+Rettungsmedizin umbenannt hat. Professor Dick und die Gründungsmitglieder schafften mit Notfall + Rettungsmedizin, wie sie in Ihrem Editorial schreiben [1], eine Zeitschrift, die es in dieser Form bisher nicht gab, denn sie war sowohl wissenschaftlich als auch praktisch orientiert und bietet für den „.... Notarzt im Rettungsdienst und den Notaufnahmearzt in der Klinik ... gemeinsam mit ihren Teams aus Rettungsassistenten und Funktionspersonal der Notaufnahme eine Fortbildung vor allem in der Diagnostik und Therapie lebensbedrohlicher Notfälle im Erwachsenen- und Kindesalter ..." und das
„... zum Wohl der uns anvertrauten Patienten."

Mit einem Anästhesiologen, einem Internisten und einem Pädiater im Schriftleiterboard wollten die Gründungsmitglieder Dick, Schuster und Lemburg ganz bewusst das Zeichen setzen, dass die Notfallmedizin interdisziplinär ausgerichtet ist.

Fast ein Jahrzehnt hat Professor Dick als Schriftleiter Notfall + Rettungsmedizin gestaltet und die Zeitschrift mit seinem hochengagierten Profil geprägt.

Und nicht nur in "unserer" Zeitschrift hat Professor Dick sich eingebracht und seine Vision der Notfallmedizin weitergetragen. Auch im Herausgeberboard der Zeitschrift Der Anaesthesist (ebenfalls Springer-Verlag) war er ab 1984 aktiv. Mit der

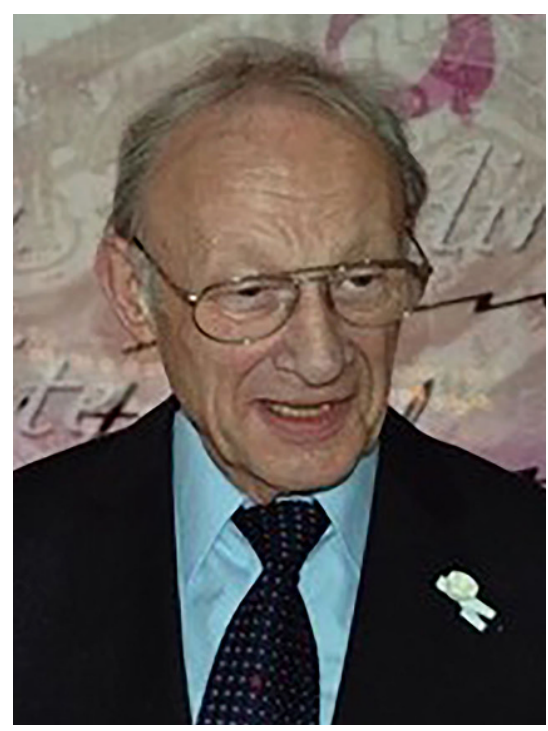

Abb. 1 ム Prof. Dr. Dr. h.c. Wolfgang F. Dick. (๑ privat, mit freundlicher Genehmigung) 
Einführung der Rubriken in dieser Zeitschrift 1992 hat er von Beginn an - erst allein und später mit Unterstützung - bis zu seinem Ausscheiden 2004 die Rubrik Notfallmedizin betreut.

Die Karriere von Professor Dick startet mit einem Studium der Theaterwissenschaften, Germanistik und Kunstgeschichte. Erst in einem zweiten Anlauf wendet er sich der Humanmedizin zu, in der er 1963 promoviert und zunächst als Assistent am Institut für Anästhesiologie der Universität Mainz arbeitet. Nach Auslandsaufenthalten und Habilitation (1970) beginnt er 1971 am Universitätsklinikum Ulm, wo er eng mit Professor Friedrich Wilhelm Ahnefeld zusammenarbeitet und dort das erste Department für Anästhesiologie in Deutschland aufbaut. 1973 folgt dann die Arbeit als Dekan. 1978 wird Professor Dick zum ärztlichen Direktor gewählt und 1982 zum Ordinarius für Anästhesiologie an der Universität Ulm ernannt.

Im Jahr 1983 erhält Professor Dick den Ruf an den angesehenen Lehrstuhl der Anästhesiologie an der Johannes GutenbergUniversität in Mainz. Auch hier übernimmt er das Amt des ärztlichen Direktors des Universitätsklinikums und zusätzlich auch Verantwortung in zahlreichen nationalen und internationalen wissenschaftlichen Gremien. So ist er von 1983 bis 1989 Sekretär der European Academy of Anaesthesiology, von 1989 bis 1991 Vizepräsident und wird im Jahr 1991 als erster Deutscher zum Präsidenten der European Academy of Anaesthesiology gewählt.

Professor Dicks zahlreiche Engagements in Zeitschriften, ganz voran in Notfall+Rettungsmedizin, in diversen Gremien und in der Klinik waren stets geprägt durch die Überzeugung, dass die Notfallmedizin nur durch einen interdisziplinären, interprofessionellen und wissenschaftlich fundierten Ansatz als Verbindung zwischen der prä- und der innerklinischen Versorgung erfolgreich sein kann.
In seinem Sinne und ihm zu Ehren werden wir dieses Ziel gemeinsam weiterverfolgen.

Bernd W. Böttiger

Christian Waydhas

Christian Wrede

Paul Herrmann

Ines Wolff

B.W. Böttiger, C. Waydhas und C. Wrede als aktuelle Schriftleitung für das Herausgeber*innenboard der Zeitschrift. P. Herrmann als Mitglied der Geschäftsleitung von Springer Medizin und Gründungsredakteur der Zeitschrift und I. Wolff als aktuelle Managing Editor

\section{Korrespondenzadresse}

\section{Dr. Ines Wolff}

Fachzeitschriften Medizin, Springer Medizin Verlag $\mathrm{GmbH}$

Tiergartenstr. 17, 69121 Heidelberg,

Deutschland

ines.wolff@springer.com

\section{Literatur}

1. DickWF, Lemburg P, Schuster H-P (1997) Eine neue Zeitschrift stellt sich vor. Notf Rettmed 0:1-2 